\title{
La enfermedad diverticular hoy. Revisión de la evidencia
}

\author{
Carolina Martínez-Pérez ${ }^{1}$, María José García-Coret ${ }^{1}$ y Francisco L. Villalba-Ferrer ${ }^{1}$
}

${ }^{1}$ Hospital General y Universitario. Valencia, España.

Recibido 2020-06-03 y aceptado 2020-10-20

Correspondencia a: Dra. Carolina Martínez-Pérez. carolinamartinezperez@ gmail.com

\section{Diverticular disease today. Evidence review}

The diverticular disease is a prevalent condition with a great economic and medical repercussion. Despite the multiple guidelines available to protocolize diagnosis and treatment, there is not unanimity in its management. We have carried out an updated review with the aim of analyzing new studies of the disease, to manage it properly and to carry out the most appropriate treatment at each time. Diverticular disease has an inherited component $(40 \%)$ and it is directly related to low fiber diet, obesity, consumption of red meat, inactivity, alcohol and NSAIDs. Due to its nonspecific symptoms, it is difficult to make a differential diagnosis. Ultrasound and abdominal CT are appropriate methods for diagnosis and early colonoscopy is recommended (4th-8th week) after acute symptoms. The most followed classification is the Hinchey Score. There is no clear evidence of the superiority of any drug in the treatment of symptomatic diverticulosis. Acute uncomplicated diverticulitis can be managed on an outpatient and the use of antibiotics is not necessary in patients without risk factors. Conservative management tends to be used in complicated diverticulitis, although in Hinchey III and IV the treatment is surgical, recommending resection of the affected area and, if possible, anastomosis with or without a protective stoma. Washing and draining is not recommended in the Hinchey III. Treatment must be agreed on an individual basis since surgical treatment is not recommended due to the number of recurrences or the age of the patient.

Key words: diverticular disease; acute diverticulitis.

\section{Resumen}

La enfermedad diverticular es muy prevalente con gran repercusión económica y médica. A pesar de las múltiples guías para protocolizar el diagnóstico y tratamiento no existe unanimidad en su manejo. Hemos realizado una revisión actualizada con el objetivo de analizar los nuevos estudios de esta enfermedad, para manejarla adecuadamente y realizar el tratamiento más adecuado en cada momento. La enfermedad diverticular tiene un componente hereditario $(40 \%)$ y presenta una relación directa con la dieta pobre en fibra, la obesidad, el consumo de carne roja, la inactividad, el alcohol y los AINEs. Por su clínica inespecífica, es difícil realizar un diagnóstico diferencial. La ecografía y el TC abdominal son métodos apropiados para el diagnóstico y se recomienda una colonoscopia de manera precoz ( $4^{\mathrm{a}}-8^{\mathrm{a}}$ semana) tras el cuadro agudo. La clasificación más seguida es la de Hinchey. En el tratamiento médico de la diverticulosis sintomática no se ha demostrado evidencia clara de ningún medicamento. La diverticulitis aguda no complicada se puede manejar ambulatoriamente y no es necesario el uso de antibióticos en pacientes sin factores de riesgo. En la diverticulitis complicada se tiende a un manejo conservador, aunque en el Hinchey III y IV el tratamiento es quirúrgico, recomendando la resección de la zona afecta y si es posible anastomosis con o sin estoma de protección. No se recomienda el lavado y drenaje en el Hinchey III. Hay que consensuar tratamiento de forma individualizada ya que no se recomienda tratamiento quirúrgico por el número de recurrencias ni por edad del paciente.

Palabras clave: enfermedad diverticular; diverticulitis; diverticulosis. 


\begin{tabular}{|c|c|}
\hline \multicolumn{2}{|l|}{ Abreviaturas } \\
\hline ED & enfermedad diverticular \\
\hline EDSNC & $\begin{array}{l}\text { enfermedad diverticular sintomática no } \\
\text { complicada }\end{array}$ \\
\hline DAC & diverticulitis aguda complicada \\
\hline DANC & diverticulitis aguda no complicada \\
\hline CSAD & $\begin{array}{l}\text { colitis segmentaria asociada con diver- } \\
\text { ticulosis }\end{array}$ \\
\hline AINES & antiinflamatorios no esteroideos \\
\hline EPOC & $\begin{array}{l}\text { enfermedad pulmonar obstructiva } \\
\text { crónica }\end{array}$ \\
\hline DGVS/DGVA & $\begin{array}{l}\text { Asociación Alemana de Gastroenterolo- } \\
\text { gía y Cirugía General y Visceral }\end{array}$ \\
\hline PCR & proteína $\mathrm{C}$ reactiva \\
\hline TC & tomografía computarizada \\
\hline US & ecografía \\
\hline RM & resonancia magnética \\
\hline DICA & $\begin{array}{l}\text { evaluación de inflamación y complica- } \\
\text { ción de la diverticulosis }\end{array}$ \\
\hline AGA & $\begin{array}{l}\text { Sociedad Americana de Gastroente- } \\
\text { rología }\end{array}$ \\
\hline WSES & $\begin{array}{l}\text { Sociedad Mundial de Cirugía de Emer- } \\
\text { gencia }\end{array}$ \\
\hline
\end{tabular}

\section{Introducción}

La enfermedad diverticular (ED) tiene alta prevalencia en la población, afectando al $65 \%$ de los mayores de 65 años, y al 5\% de los menores de 40 años, conllevando una gran repercusión médica y económica. Existe una gran variabilidad clínica en su presentación, con un $80 \%$ de pacientes asintomáticos y un $20 \%$ de sintomáticos, de los cuales, el $5 \%$ presentarán cuadros clínicos graves.

La ED engloba distintas situaciones clínicas: Diverticulosis asintomática; enfermedad diverticular sintomática no complicada (EDSNC) o diverticulosis sintomática (20\%); diverticulitis aguda (DA) $(5 \%)$, DA complicada (DAC) $(15 \%)^{1}$; y la colitis segmentaria asociada con diverticulosis (CSAD) (0,3\%-1,3\%). La CSAD es una entidad recientemente estudiada como una manifestación poco conocida de la ED con una inflamación crónica segmentaria del colon en pacientes con diverticulosis que simula clínica, endoscópica e histológicamente una enfermedad inflamatoria intestinal (EII), siendo muchas veces indistinguibles, siendo necesarias biopsias para la exclusión de la inflamación en el recto y otras áreas del colon para diferenciarlas.

Llama la atención las numerosas guías que existen de la ED, que pretenden protocolizar, estanda- rizar el diagnóstico y las estrategias terapéuticas de una manera razonable, pero no existen recomendaciones claras ni unánimes.

El objetivo de esta revisión es conocer los avances que se producen en la ED, para manejarla adecuadamente, evitar su progresión y recurrencia, así como realizar el tratamiento más adecuado en cada fase.

\section{Metodología}

Dos autores diferentes (CMP Y MJGC) llevaron a cabo una revisión de la literatura utilizando las palabras clave "acute diverticulitis, diverticular disease, diverticulosis" en la base de datos Medline de artículos publicados en inglés y español entre 1990 y 2019. Se identificaron 211 artículos, de los que se incluyeron 40 en esta revisión. Los criterios de inclusión fueron: guías de práctica clínica, estudios clínicos controlados, estudios de cohorte, metaanálisis y revisiones sistemáticas. Se ha utilizado la clasificación de las recomendaciones en función del nivel de evidencia (NE) disponible de la Agency for Healthcare Research and Quality ${ }^{2}$.

\section{Factores de riesgo}

Se estima un componente hereditario de la enfermedad diverticular en el $40 \%$ de los pacientes y se ha visto un polimorfismo del gen TFNSF15 como marcador de la DAC. Más recientemente, una rara variante del gen laminina- $\beta 4$ (LAMB4) se ha asociado con diverticulitis de inicio temprano y diverticulitis esporádica no familiar ${ }^{3}$.

Existe un sobrecrecimiento en la flora intestinal de la enfermedad diverticular sintomática no complicada (EDSNC) y de diverticulitis aguda no complicada (DANC) ${ }^{4}$. La dieta pobre en fibra se asocia a cambio en la flora bacteriana y alteración de la inmunidad local, provocando una inflamación crónica de bajo grado que podría predisponer a la diverticulitis aguda (DA).

Otros factores relacionados con la aparición de diverticulosis, son las dietas ricas en carne roja, la obesidad por el efecto proinflamatorio de las adipocinas y las quimiocinas, el tabaquismo, la inactividad física, el alcohol y los antiinflamatorios no esteroideos (AINES) ${ }^{5}$.

La insuficiencia renal crónica, la enfermedad pulmonar obstructiva crónica (EPOC), la diabetes, la vasculitis y las drogas inmunosupresoras están relacionadas con una evolución más severa de la diverticulitis y se deben tener en cuenta en las estrategias terapéuticas. Hay autores (DGVS/DGVA) que 
encuentran una mayor tasa de diverticulitis aguda complicada (DAC) en pacientes que toman morfina crónicamente ${ }^{6}$.

\section{Clínica y diagnóstico}

La clínica de la EDSNC es leve e inespecífica, lo más frecuente es el dolor abdominal, meteorismo y cambios en los hábitos intestinales. Hay que hacer un diagnóstico diferencial, sobre todo, con el síndrome de intestino irritable, donde el dolor abdominal es más difuso ${ }^{7}$.

El diagnóstico de diverticulosis puede establecerse mediante enema opaco, colonoscopia o incluso tomografía computarizada (TC); si bien, una mayoría de pacientes serán diagnosticados de enfermedad diverticular durante el primer episodio de DA.

La hemorragia diverticular es una complicación rara, caracterizada por rectorragia indolora y generalmente autolimitada, siendo la colonoscopia la técnica indicada para su diagnóstico ${ }^{8}$.

La presentación de la DA puede variar desde un dolor abdominal leve por perforación contenida, hasta un dolor abdominal intenso con irritación peritoneal por una perforación libre. La tríada de dolor abdominal en cuadrante inferior izquierdo, ausencia de vómitos y PCR (proteína $\mathrm{C}$ reactiva) $>5 \mathrm{mg} / \mathrm{dl}$, tiene una sensibilidad del $97 \%$ para el diagnóstico de la $\mathrm{DA}^{6}$

En ocasiones, los episodios recurrentes de DA pueden evolucionar a una fístula colovesical o colovaginal, una estenosis y oclusión.

En la DA la TC y la ecografía (US) se consideran los métodos apropiados para el diagnóstico, con sensibilidades comparables mayores del 90\%. En estudios prospectivos, la US alcanza una sensibilidad y especificidad del $91 \%$ y $96 \%{ }^{9}$. La TC identificó perforaciones cubiertas y abscesos con eficacia del 96\% (sensibilidad: 100\% y especificidad: 91\%), además, ayudó a realizar un diagnóstico diferencial con el cáncer de colon, siendo el error en este diag-

Tabla 1. Clasificación de Hinchey modificada

\begin{tabular}{|lll|}
\hline $\begin{array}{l}\text { Diverticulitis aguda } \\
\text { no complicada }\end{array}$ & Grado 0 & Hallazgos \\
$\begin{array}{lll}\text { Diverticulitis aguda } \\
\text { complicada }\end{array}$ & Grado Ib & Flemón-inflamación pericólica \\
& Grado II & $\begin{array}{l}\text { Absceso pericólico o mesocólico }<5 \mathrm{~cm} \\
\text { neal o absceso a distancia de la zona inflamada }\end{array}$ \\
& Grado III & Peritonitis purulenta generalizada \\
& Grado IV & Peritonitis fecaloidea generalizada \\
\hline
\end{tabular}

nóstico diferencial del 5\%. La resonancia magnética (RM) no está recomendada en el estudio diagnóstico de primera línea, aunque podría ser utilizada en pacientes embarazadas con ecografía no concluyente.

En los cuadros clínicos claramente compatibles con DANC hay autores que recomiendan un tratamiento empírico sin TC, aunque otros recomiendan realizarlo de inicio para una correcta estadificación, en los casos con empeoramiento clínico, dudas diagnósticas y ante la sospecha de que sea una DAC $(\mathrm{NE} \text { IV })^{10}$.

Existe un 11,4\% de cánceres que imitan el absceso diverticular. Únicamente un estudio holandés ${ }^{11}$ defiende la realización de una colonoscopia en la fase aguda si existe alta sospecha de EII, colitis isquémica o neoplasia. Sin embargo, mayoritariamente recomiendan una colonoscopia rutinaria precoz entre la $4^{\mathrm{a}}$ y $8^{\mathrm{a}}$ semanas tras la DA, sobre todo tras DAC o en pacientes ancianos, para excluir el diagnóstico erróneo con una neoplasia colónica (NE IV) ${ }^{6,8,12}$.

\section{Clasificación}

No existe un consenso a la hora de clasificar el grado de diverticulitis, sin embargo, la mayoría de las guías utilizan la clasificación de Hinchey porque permite adaptarse mejor al diagnóstico obtenido por TC y ajustar la orientación terapéutica. Sí existe consenso a la hora de distinguir entre diverticulitis no complicada (Hinchey 0-Ia) y complicada (Hinchey Ib-II-III-IV) (Tabla 1).

Recientemente, se ha propuesto una clasificación endoscópica para evaluar la inflamación y la complicación diverticular (DICA) que toma en consideración varios ítems puntuados y subítems, clasificándolo en DICA 1, DICA 2 y DICA 3. Al ser tan reciente hay pocos estudios que lo utilicen, pero parece que los pacientes con DICA 2 se benefician de un tratamiento programado para prevenir la aparición y recurrencia de la $\mathrm{DA}^{13}$.

\section{Tratamiento (Tabla 2)}

\section{Tratamiento conservador}

La diverticulosis asintomática se considera un hallazgo incidental, no requiere tratamiento ni seguimiento. Las recomendaciones, aunque con baja calidad de evidencia (NE IC), son una dieta rica en fibra, dejar de fumar, evitar el consumo de alcohol y realizar ejercicio ${ }^{8,14-16}$. La diabetes no controlada y la hipovitaminosis D se relaciona con mayor progresión a EDSNC y DA, por lo que hay que tratarlas ${ }^{17}$.

En la EDSNC además de las recomendaciones dietéticas podríamos sumar el uso de algunos fármacos. Con la rifaximina (400 mg 7 días al mes) se han 
encontrado diferencias estadísticamente significativas en un metaanálisis, recomendando su uso (NE Ia). Con respecto a la mesalazina ${ }^{14}$ (NE IIb) y los probióticos $^{18}$ (NE IV) no existe suficiente evidencia que avale su recomendación.

En la CSAD no hay evidencia suficiente para recomendar ningún tratamiento, sin embargo, en base a su fisiopatología se ha sugerido la utilización de mesalazina en combinación o no con esteroides ${ }^{19}$ (NE Ib).

Para el tratamiento de la DANC (Hinchey 0 y Ia) se recomienda un tratamiento sintomático ambulatorio. Hay estudios prospectivos randomizados, caso control e incluso una revisión de la Cochrane que demuestran que el uso de antibióticos no influye en la recurrencia, ni en la aparición de complicaciones, tasas de ingreso o mortalidad ${ }^{20-22}$ (NE Ib). Los antibióticos sólo deben usarse en casos seleccionados como son los pacientes con signos de infección generalizada, inmunodeprimidos o si existe un fracaso del tratamiento conservador sin antibióticos ${ }^{23}$ (NE Ia). No hay consenso en la dieta que debe seguir el paciente, aunque la mayoría recomienda dietas pobres en residuos durante el episodio agudo.

Para recomendar un tratamiento ambulatorio es importante un correcto diagnóstico, estadificación y selección de los pacientes. Es posible, si el paciente tiene un buen estado general, trénsito intestinal, entorno familiar adecuado y puede acudir a las revisio$\operatorname{nes}^{24}$ (NE Ib). No se recomienda el tratamiento ambulatorio en mayores de 85 años, ni con factores de riesgo de mala evolución (como son los antecedentes de perforación por AINEs, inmunosupresión por enfermedad o inducida) y tampoco se recomienda si existe sintomatología persistente o empeoramiento clínico. En estos pacientes, lo recomendable sería un tratamiento hospitalario con antibióticos ${ }^{25}$ (NE $\mathrm{Ib}$ ), aunque no existe consenso en la vía de administración ni pautas.

Las DAC con Hinchey Ib, II se deben tratar con ingreso hospitalario y antibióticos ${ }^{26}$. No existe consenso en la mejor vía de administración de los antibióticos $^{6,11,24}$, ni en el número de días que hay que mantener el tratamiento. Según las recomendaciones

Tabla 2. Tratamiento de la enfermedad diverticular

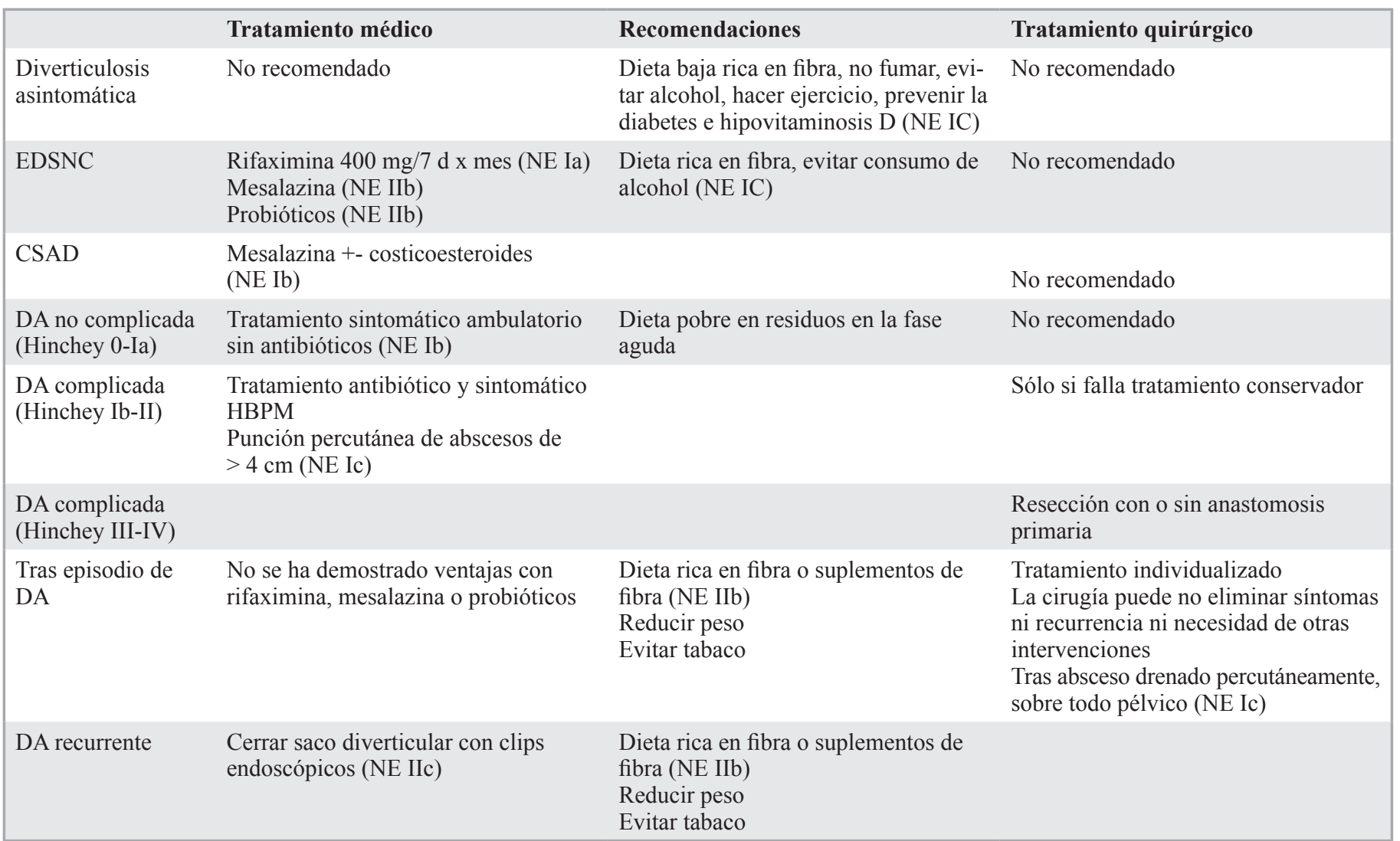


holandesas ${ }^{11}, 4$ días de antibióticos intravenosos son tan efectivos como 7 días (NE IIa). Tampoco existen pautas claras del antibiótico o combinación de elección.

A todos los pacientes se les debe administrar profilaxis de heparina de bajo peso molecular.

Durante las primeras $48 \mathrm{~h}$ el paciente requiere un estrecho control clínico, para poder modificar la actitud terapéutica si fuera necesario.

Respecto al manejo de los abscesos, el tratamiento conservador con antibióticos de amplio espectro tiene una tasa de éxito del $70 \%$ en abscesos menores de $4 \mathrm{~cm}^{27}$ (NE Ic). Se recomienda realizar un drenaje percutáneo para los fracasos del tratamiento médico y de entrada en los abscesos más grandes $(>3-5 \mathrm{~cm})$ con una tasa de éxito hasta del $80 \%{ }^{28}$ (NE Ic). La cirugía debe planificarse cuando falla el tratamiento conservador.

Tras el episodio de DA la Sociedad Americana de Gastroenterología (AGA) recomienda una dieta rica en fibra o suplementos de fibra ${ }^{16}$ (NE IIb). No se ha podido demostrar en los diferentes estudios ninguna ventaja para el uso de la terapia con rifaximina, mesalazina, probióticos o restricciones dietéticas. La reducción de peso y el abandono del hábito tabáquico pueden reducir el riesgo de diverticulitis recurrente $^{29}$. La recurrencia de la diverticulitis aguda tras un primer episodio se estima entre el 15\% y $30 \%{ }^{30}$.

Recientemente se ha propuesto cerrar el saco diverticular por endoscopia colocando endoclips ${ }^{31}$ (NE IIc), pero es necesario un seguimiento a largo plazo para evaluar la recaída y confirmar este resultado.

\section{Tratamiento quirúrgico}

La recurrencia de la DA después de un primer episodio de diverticulitis tratado médicamente es menor del 20\%, mientras que el riesgo de recurrencia después del tratamiento quirúrgico no se elimina totalmente, siendo del $7 \%{ }^{32}$, además, tras la cirugía aumenta considerablemente el coste relacionado con la morbilidad y mortalidad.

Hasta hace pocos años las guías clínicas recomendaban realizar una cirugía electiva tras dos episodios de DANC o tras un episodio en pacientes jóvenes ${ }^{16,33}$, porque se consideraba que con cada nuevo episodio de DANC se respondía menos al tratamiento médico. Estas recomendaciones han sido muy cuestionadas y no se incluyen en las nuevas guías, incluso resaltan que el episodio más grave en la DA recurrente es el primero ${ }^{34}$, existiendo menor riesgo de recaída y menor tasa de cirugía urgente en los episodios sucesivos (NE III).

Además, la cirugía puede no suponer el punto final de la enfermedad, persistiendo los síntomas en un $27 \%-33 \%$ de los pacientes y la recurrencia entre un $1 \%-10 \%$, requiriendo nueva resección hasta en el $1 \%{ }^{35}$ (NE III).

Por lo tanto, el tratamiento debe ser individualizado teniendo en cuenta la morbilidad del paciente, los riesgos de la cirugía, la afectación del estilo de vida ocasionada por la enfermedad, la exclusión de que se trate de una neoplasia, así como la severidad de los episodios y la persistencia de los mismos. Además, el paciente debe ser conocedor de la posibilidad de tener resultados con alteraciones funcionales y persistencia de los síntomas.

Es debatido si se recomienda la resección en los pacientes inmunodeprimidos, algunos autores la recomiendan ${ }^{8,11,15,24}$ y otros no lo especifican ${ }^{6,12}$.

La DA con absceso (Hinchey Ib o II) de forma conservadora, muestra que a largo plazo el 51\% de los pacientes con absceso mesocólico y el 71\% con absceso pélvico requieren cirugía ${ }^{27}$ (NE Ic). La WSES (Sociedad Mundial de Cirugía de Emergencia) sugiere realizar una resección temprana tras absceso pélvico drenado por radiología intervencionista, porque estos pacientes se asocian con peor resultado a largo plazo, en comparación con los abscesos mesocólicos ${ }^{24}$ (NE Ic).

En las guías anteriores al 2013 se recomendaba lavado laparoscópico sin resección como terapia alternativa para la diverticulitis purulenta, Hinchey III, pero en las guías más recientes no se recomien$\mathrm{da}^{36}$ (NE Ib).

La cirugía de la diverticulitis se considera un procedimiento laparoscópico de complejidad mayor, recomendándose en la cirugía programada y existiendo discrepancia en la cirugía urgente.

Lo más recomendado en la cirugía de la diverticulitis Hinchey III y IV es la resección con anastomosis primaria, con o sin ileostomía deriva$\operatorname{tiva}^{37}$ (NE Ib). El objetivo es evitar, si se puede, la colostomía terminal ya que sólo el $57 \%$ se revertirán ${ }^{38}$. Hay que tener en cuenta, que la resección y anastomosis primaria, aunque es la opción ideal, es un procedimiento arriesgado por su elevado índice de dehiscencia anastomótica (en torno al 8\%). Se consideran contraindicaciones para la anastomosis primaria el shock séptico, la inestabilidad hemodinámica, la corticoterapia crónica y el mal estado general del paciente, la obesidad hay autores que la citan como factor predictivo independiente de la dehiscencia ${ }^{39}$.

La obstrucción secundaria a una estenosis diverticular ocurre en el $10 \%$ de los pacientes con obstrucción colónica, siendo provocada por un engrosamiento y una contracción de la pared intestinal secundaria a la inflamación crónica. Las dilataciones 
con balón y los stents están desaconsejados ${ }^{40}$, y lo más recomendado es la cirugía con anastomosis primaria si es posible ${ }^{11}$ (NE Ib).

La formación de una fístula tras diverticulitis es del $2 \%$, siendo las más frecuentes las colovesicales (50\%), seguidas de las colovaginales. Realizaremos intervención precoz de la complicación fistulosa o estenosis cuando no pueda llegarse a un diagnóstico diferencial con el cáncer.

Existen casos en los que se producen hemorragias diverticulares masivas, el tratamiento es una colonoscopia terapéutica o una embolización selectiva, reservando la cirugía cuando estos tratamientos no están disponibles o han fracasado ${ }^{31}$.

\section{Comentarios}

Continúa existiendo controversia sobre las mejores medidas a adoptar en cada situación, por lo que destacamos a continuación los aspectos actualmente con más consenso:

- Control ambulatorio, sin antibioterapia en pacientes con DANC (Hinchey 0-Ia) y sin factores de riesgo de mala evolución.

- Tratamiento intrahospitalario con antibioterapia en el resto de cuadros de DANC y DAC sin indicación de cirugía

- Drenaje percutáneo en los abscesos mayores a $3-5 \mathrm{~cm}$ de diámetro.
- Abordaje laparoscópico en cirugía programada.

- Indicación de cirugía programada individualizada tras una DA no complicada, siendo la inmunodepresión y el trasplante las comorbilidades con mayor recomendación para indicar la cirugía.

- En la DAC con absceso pélvico (Hinchey II), resuelto con tratamiento conservador, es recomendable la cirugía programada precoz mediante resección.

- En la DAC, Hinchey III y IV, es recomendable la cirugía urgente con resección.

- En la DAC con fístula colovesical o colovaginal es recomendable la cirugía programada precoz resectiva.

- En las cirugías resectivas es recomendable la anastomosis primaria, con o sin ileostomía derivativa, si no existe contraindicación.

\section{Responsabilidades éticas}

Protección de personas y animales. Los autores declaran que para esta investigación no se han realizado experimentos en seres humanos ni en animales.

Confidencialidad de los datos. Los autores declaran que en este artículo no aparecen datos de pacientes.

Conflictos de interés: no hay.

\section{Bibliografía}

1. Shahedi K, Fuller G, Bolus R, Cohen E, $\mathrm{Vu} \mathrm{M}$, Shah R, et al. Long-term risk of acute diverticulitis among patients with incidental diverticulosis found during colonoscopy. Clin Gastroenterol Hepatol. 2013;11:1609-13

2. Agency for Healthcare Research an Quality (AHRQ) EPC Evidence Reports [Internet]. Rockville: AHQR. [acceso 12/2/2009]. Disponible en http://www.ahrg.gov/clinic/ epcinfex.htm\#methodology.

3. Coble JL, Sheldon KE, Yue F, Salameh TJ, Harris LRIII, Deiling S, et al. Identification of a rare LAMB4 variant associated with familial diverticulitis through exome sequencing. Hum Mol Genet. 2017;26:3212-20.

4. Tursi A, Brandimarte G, Giorgetti GM, Elisei W. Assessment of small intestinal bacterial overgrowth in uncomplicated acute diverticulitis of the colon. World $\mathrm{J}$ Gastroenterol. 2005;11:2773-6.

5. Strate LL, Liu YL, Huang ES, Giovannucci EL, Chan AT. Use of aspirin or nonsteroidal anti-inflammatory drugs increases risk for diverticulitis and diverticular bleeding. Gastroenterology 2011;140:1427-33.

6. Leifeld L, Germer CT, Bohm S, Dumoulin FL, Hauser W, Kreis M, et al. [S2k guidelines diverticular disease/diverticulitis]. Z Gastroenterol. 2014;52:663-710.

7. Lanas A, Abad-Baroja D, Lanas-Gimeno A. Progress and challenges in the management of diverticular disease: which treatment? Therap Adv Gastroenterol. 2018;11:1-21.

8. Pietrzak A, Bartnik W, Szczepkowski M, Krokowicz P, Dziki A, Regula J, et al. Polish interdisciplinary consensus on diagnostics and treatment of colonic diverticulosis (2015). Pol Przegl Chir. 2015;87:203-20.

9. King WC, Shuaib W, Vijayasarathi A, Fajardo CG, Cabrera WE, Costa JL. Benefits of sonography in diagnosing suspected uncomplicated acute diverticulitis. J Ultrasound Med. 2015;34:53-8.

10. Sartelli M, Moore FA, Ansaloni L, Di Saverio S, Coccolini F, Griffiths EA, et al. A proposal for a $\mathrm{CT}$ driven classification of left colon acute diverticulitis. World J Emerg Surg. 2015;10:1-11.

11. Andeweg CS, Mulder IM, Felt-Bersma RJF, Verbon A, van der Wilt GJ, van Goor $\mathrm{H}$, et al. Guidelines of diagnostics and treatment of acute left-sided colonic diverticulitis. Dig Surg. 2013;30:278-92.

12. Binda GA, Cuomo R, Laghi A, Nascimbeni R, Serventi A, Bellini D, et al. Practice parameters for the treatment of colonic diverticular disease: Italian 
Society of Colon and Rectal Surgery (SICCR) guidelines. Tech Coloproctol. 2015;19:615-26.

13. Štimac D, Nardone G, Mazzari A, Crucitti A, Maconi G, Elisei W, et al. Diverticular Disease of the Colon What's New in Diagnosing Diverticular Disease 2019;28:17-21.

14. Cuomo R, Barbara G, Pace F, Annese V, Bassotti G, Binda GA, et al. Italian consensus conference for colonic diverticulosis and diverticular disease. United Eur Gastroenterol J. 2014;2:41342.

15. Andersen JC, Bundgaard L, Elbrond H, Laurberg S, Walker LR, Stovring J. Danish national guidelines for treatment of diverticular disease. Dan Med J. 2012;59:C4453.

16. Stollman N, Smalley W, Hirano I, Adams MA, Dorn SD, Dudley-Brown SL, et al. American Gastroenterological Association Institute Guideline on the Management of Acute Diverticulitis. Gastroenterology 2015;149:1944-9.

17. Tursi A, Elisei W, Picchio M, Di Mario F, Brandimarte G. Serum levels of vitamin $\mathrm{D}$ are associated with the severity of the endoscopic appearance of diverticular disease of the colon according to DICA classification. J Gastrointestin Liver Dis. 2016;25:567-8.

18. Lahner E, Bellisario C, Hassan C, Zullo A, Esposito G, Annibale B. Probiotics in the Treatment of Diverticular Disease. A Systematic Review. J Gastrointestin Liver Dis. 2016;25:79-86.

19. Kucejko RJ, Poggio JL. Considerations and Changes in the Evaluation, Management, and Outcomes in the Management of Diverticular Disease: The Diagnosis, Pathology and Treatment of Diverticular Colitis 2018;1:1-5.

20. Daniels L, Unlu C, de Korte N, van Dieren S, Stockmann HB, Vrouenraets BC, et al. Randomized clinical trial of observational versus antibiotic treatment for a first episode of CT-proven uncomplicated acute diverticulitis. Br J Surg. 2017;104:52-61.

21. Mege D, Yeo H. Meta-analyses of Current Strategies to Treat Uncomplicated
Diverticulitis. Dis Colon Rectum 2019;62:371-8.

22. Shabanzadeh DM, Wille-Jorgensen P. Antibiotics for uncomplicated diverticulitis. Cochrane Database Syst Rev. 2012;11:CD009092.

23. Emile SH, Elfeki H, Sakr A, Shalaby M. Management of acute uncomplicated diverticulitis without antibiotics: a systematic review, meta-analysis, and meta-regression of predictors of treatment failure. Tech Coloproctol. 2018;22:499509.

24. Sartelli M, Catena F, Ansaloni L, Coccolini F, Griffiths EA, Abu-Zidan FM, et al. WSES Guidelines for the management of acute left sided colonic diverticulitis in the emergency setting. World J Emerg Surg. 2016;11:1-15.

25. Sánchez-Velázquez P, Grande L, Pera M. Outpatient treatment of uncomplicated diverticulitis: a systematic review. Eur J Gastroenterol Hepatol. 2016;28:622-7.

26. Chua TC, Jeyakumar A, Ip JCY, Yuide PJ, Burstow MJ. Conservative management of acute perforated diverticulitis: A systematic review. J Dig Dis. 2020;21:638.

27. Ambrosetti $\mathrm{P}$, Chautems R, Soravia C, Peiris-Waser N, Terrier F. Longterm outcome of mesocolic and pelvic diverticular abscesses of the left colon: a prospective study of 73 cases. Dis Colon Rectum 2005;48:787-91.

28. Binda GA, Arezzo A, Serventi A, Bonelli L, Facchini M, Prandi M, et al. Multicentre observational study of the natural history of left-sided acute diverticulitis. Br J Surg. 2012;99:276-85.

29. Hjern F, Wolk A, Hakansson N. Smoking and the risk of diverticular disease in women. Br J Surg. 2011;98:997-1002.

30. Broderick-Villa G, Burchette RJ, Collins JC, Abbas MA, Haigh PI, Wolff BG, et al. Hospitalization for acute diverticulitis does not mandate routine elective colectomy. Arch Surg. 2005;140:576-83.

31. Zullo A, Gatta L, Vassallo R, Francesco V De, Manta R, Monica F. Paradigm shift: the Copernican revolution in diverticular disease 2019:541-53.
32. López-Borao J, Kreisler E, Millan M, Trenti L, Jaurrieta E, Rodríguez-Moranta $\mathrm{F}$, et al. Impact of age on recurrence and severity of left colonic diverticulitis. Colorectal Dis. 2012;14:e407-12.

33. Kohler L, Sauerland S, Neugebauer E. Diagnosis and treatment of diverticular disease: results of a consensus development conference. The Scientific Committee of the European Association for Endoscopic Surgery. Surg Endosc. 1999;13:430-6.

34. Pittet O, Kotzampassakis N, Schmidt S, Denys A, Demartines N, Calmes J-M. Recurrent left colonic diverticulitis episodes: more severe than the initial diverticulitis? World J Surg. 2009;33:54752 .

35. Janes S, Meagher A, Frizelle FA. Elective surgery after acute diverticulitis. Br J Surg. 2005;92:133-42.

36. Schultz JK, Yaqub S, Wallon C, Blecic L, Forsmo HM, Folkesson J, et al. Laparoscopic Lavage vs Primary Resection for Acute Perforated Diverticulitis: The SCANDIV Randomized Clinical Trial. JAMA 2015;314:1364-75.

37. Benson AB, Venook AP, Al-Hawary MM, Cederquist L, Chen YJ, Ciombor KK, et al. Rectal cancer, version 2.2018 clinical practice guidelines in Oncology. JNCCN J Natl Compr Cancer Netw. 2018;16:874901.

38. van de Wall BJM, Draaisma WA, Schouten ES, Broeders IAMJ, Consten ECJ. Conventional and laparoscopic reversal of the hartmann procedure: A review of literature. J Gastrointest Surg. 2010;14:743-52.

39. Biondo S, Pares D, Kreisler E, Rague JM, Fraccalvieri D, Ruiz AG, et al. Anastomotic dehiscence after resection and primary anastomosis in left-sided colonic emergencies. Dis Colon Rectum 2005;48:2272-80.

40. Meisner S, Hensler M, Knop FK, West F, Wille-Jorgensen P. Self-expanding metal stents for colonic obstruction: experiences from 104 procedures in a single center. Dis Colon Rectum 2004;47:444-50. 